Nicole Kaiser

\title{
Prix SEVAL 2020: Laudatio
}

Beitragsart: Mitteilungen

Zitiervorschlag: Nicole Kaiser, Prix SEVAL 2020: Laudatio, in: LeGes 31 (2020) 3 
[1] Der Prix SEVAL 2020 wurde dem Forschungsteam Dr. Céline Mavrot, Dr. Susanne Hadorn und Prof. Dr. Fritz Sager für ihren wissenschaftlichen Artikel «Mapping the Mix: Linking instruments, settings and target groups in the study of policy mixes» (Mavrot, Hadorn, Sager 2019) verliehen. Die Autorinnen sind Postdoktorandinnen, der Autor ordentlicher Professor am Kompetenzzentrum für Public Management (KPM) der Universität Bern.

[2] Beim Artikel handelt es sich um ein wissenschaftliches Produkt aus einem kürzlich abgeschlossenen umfangreichen Evaluationsprojekt, bei dem die Forscherinnen und Forscher im Auftrag des Tabakpräventionsfonds 14 kantonale Tabakpräventionsprogramme evaluiert sowie einen kantonsübergreifenden Evaluationsbericht verfasst haben.

[3] Der Artikel schlägt ein theoretisches Modell vor, um Policy Mixes und ihre Wirkungen zu untersuchen. Dabei ergänzen die Autorinnen und Autoren das bisherige theoretische Modell, welches die verschiedenen Politikinstrumente und deren Zusammenspiel umfasst, um den Kontext. Unter Kontext meinen sie erstens das Setting, in dem ein Politikinstrument umgesetzt wird, und zweitens die Zielgruppe eines Politikinstruments. Dazu ein Beispiel: Bei einer NichtraucherKampagne an Sekundarschulen ist die Sekundarschule das Setting, die Jugendlichen sind die Zielgruppe. Dadurch schärft der Artikel unseren Blick für denjenigen Teil des Policy-Prozesses, der sich auf der Ebene der Menschen und ihrem Umfeld - den «Empfängern» von Politikinstrumenten - abspielt.

[4] Der Artikel hat die Jury des Prix SEVAL bezüglich aller vier Beurteilungskriterien überzeugt.

[5] Erstens überzeugt der Artikel durch seine hohe wissenschaftliche Qualität. Der Artikel ist in der hochrangingen Zeitschrift «Research Policy» erschienen und stellt die Weiterentwicklung des theoretischen Policy-Mix-Modells ins Zentrum. Dabei stützen sich die Autorinnen und Autoren auf den Ansatz der realistic evaluation (Pawson/Trlley 1997), welcher den Kontext und den Outcome eines Politikinstruments kausal miteinander in Verbindung setzt. Auch methodisch überzeugt der Artikel. Es wird ein mixed-method-Ansatz verfolgt. Dabei stützt sich der Artikel auf eine beeindruckend grosse Datengrundlage aus den Evaluationen der kantonalen Tabakpräventionsprogramme, die Dokumentenanalysen, Selbstevaluationsberichte, Interviews und Kontextanalysen umfassten.

[6] Zweitens ist die Studie auf mindestens zwei Ebenen originell. Der Artikel bringt ein weiterentwickeltes Policy-Mix-Modell hervor, das neu die Settings und Zielgruppen miteinbezieht. Zudem erhalten verschiedene Evaluationskriterien, so z.B. die Kohärenz, eine präzisere Bedeutung. So kann nicht nur die Kohärenz zwischen verschiedenen Politikinstrumenten eines Policy-Mix bestimmt werden, sondern auch die Kohärenz zwischen den Politikinstrumenten, ihren Settings und ihren Zielgruppen. Dazu ein Beispiel zweier Politikinstrumente eines Policy-Mix: An Sekundarschulen findet eine Nichtraucher-Kampagne statt. Gleichzeitig gibt es Beratungsangebote von Freizeitorganisationen, um Jugendliche dazu zu bewegen, mit dem Rauchen aufzuhören. Zwar werden die Raucherinnen und Raucher unter den Jugendlichen mittels der Kampagne informiert, sie haben aber keinen Zugang zum Beratungsangebot, um mit dem Rauchen aufzuhören. Gleichzeitig werden die Beratungsangebote kaum von den Jugendlichen in Anspruch genommen. In diesem Beispiel ist der Policy-Mix zwar kohärent in Bezug auf seine Politikinstrumente, nicht aber in Bezug auf seine Settings - was schlussendlich die Wirkung des Policy-Mix beeinträchtigt. [7] Zum dritten ist die Studie auf verschiedene Art und Weise relevant für die Evaluationspraxis. Der Artikel zeigt auf, dass Policies wirksamer sind, wenn das Zusammenspiel von Instrument, Setting und Zielgruppen berücksichtigt wird. Die Autorinnen und Autoren schlagen vor, diese Erkenntnis sowohl in die Planung von Politikinstrumenten wie auch in die Planung von Evalua- 
tionen zu integrieren. Auch wenn dies aus Sicht der Evaluationspraxis bereits bekannt vorkommt; der Artikel liefert eine theoretische Grundlage dafür. Zudem zeigt der Artikel auf, dass innerhalb von Policy-Mixes die ständige Koordination zwischen den verschiedenen beteiligten Akteuren, so «Sender» der Politikinstrumente, umsetzende Akteure je Setting und «Empfänger» der Politikinstrumente, wichtig ist für die Wirkung des gesamten Policy-Mix.

[8] Nicht zuletzt entspricht der Artikel formalen wissenschaftlichen Kriterien. Er ist klar strukturiert, angenehm zu lesen und für Evaluatorinnen und Evaluatoren eine Lektüre wert.

[9] Die Jury freut sich, den Prix SEVAL 2020 an das Forschungsteam Dr. CÉline Mavrot, Dr. Susanne Hadorn und Prof. Dr. Fritz Sager zu überreichen. Die SEVAL anerkennt damit, dass die Autorinnen und Autoren einen hervorragenden Beitrag zur Weiterentwicklung der Evaluationstheorie geleistet haben.

\section{Ausschreibung des Prix SEVAL 2021}

[10] Um die wissenschaftliche Auseinandersetzung mit Evaluation zu fördern hat die SEVAL den Prix SEVAL geschaffen. Mit diesem Preis werden einmal jährlich wissenschaftliche Arbeiten ausgezeichnet, die einen hervorragenden Beitrag zur Weiterentwicklung von Theorie und Praxis der Evaluation oder zur Verwendung von Evaluation liefern.

[11] Der Prix SEVAL wird jeweils anlässlich des SEVAL-Jahreskongresses verliehen. Der Preis ist mit CHF 3000.- dotiert und beinhaltet auch die Veröffentlichung eines Beitrages in LeGes.

[12] Teilnahmeberechtigt sind Qualifikationsarbeiten aller Stufen, in Fachzeitschriften publizierte Artikel, Fachbücher oder Evaluationsstudien von Studierenden, Forschenden und anderen wissenschaftlich tätigen Personen in der Schweiz, die einen Bezug zur Evaluation aufweisen. Arbeiten können ab sofort beim SEVAL-Sekretariat eingereicht werden (secretariat@seval.ch), Einsendeschluss ist der 2. April 2021. Sie können auf Deutsch, Französisch, Italienisch oder Englisch verfasst sein. Die eingereichten Arbeiten werden auf ihre Wissenschaftlichkeit, Originalität, Praxisrelevanz und Form beurteilt.

[13] Die Jury für den Prix SEVAL setzt sich wie folgt zusammen:

- Prof. Pia Gabriel-Schärer, Hochschule Luzern, Soziale Arbeit, Luzern (Präsidentin der Jury)

- Prof. Pirmin Bundi, IDHEAP Universität Lausanne

- Marion Baud-Lavigne, Evaluatorin, Parlamentarische Verwaltungskontrolle

- Nicole Kaiser, Senior Projektleiterin, econcept AG

[14] Die offizielle Ausschreibung, das detaillierte Reglement, sowie weitere Informationen zum Prix SEVAL sind auf der SEVAL-Webseite (https://www.seval.ch/publikationen-prix-seval/prixseval/) aufgeschaltet.

\section{Annonce du Prix SEVAL 2021}

[15] Pour encourager les discussions scientifiques sur le thème des évaluations, la SEVAL a créé le Prix SEVAL. Le prix est décerné à des travaux scientifiques qui contribuent d'une manière remarquable au développement de théories et de pratiques, ou pour l'utilisation d'évaluations. 
[16] Le Prix SEVAL est remis une fois par année, lors du congrès annuel de la SEVAL. Il est honoré par un montant de CHF 3000.- et prévoit par ailleurs la publication d'un article dans LeGes.

[17] Sont admis les travaux académiques de tous les niveaux, des articles publiés dans des revues scientifiques, des livres scientifiques ou des études d'évaluation d'étudiants, de chercheurs ou d'autres scientifiques en Suisse avec un lien à l'évaluation. Les travaux mis au concours peuvent dès à présent être remis au secrétariat de la SEVAL (secretariat@seval.ch). Délai : le 2 avril 2021.

[18] Les travaux soumis peuvent être rédigés en français, allemand, italien ou anglais. Ils seront considérés selon leur caractère scientifique, leur originalité, leur intérêt pratique et leur forme. Le jury pour le Prix SEVAL est composé des personnes suivantes :

- Prof. Pia Gabriel-Schärer, Haute école Lucerne, Travail social, Lucerne

- Prof. Pirmin Bundi, IDHEAP Université de Lausanne

- Marion Baud-Lavigne, Evaluatrice, Contrôle parlementaire de l'administration

- Nicole Kaiser, responsable de projet senior, econcept AG

[19] L’annonce officielle, le règlement détaillé ainsi que des informations supplémentaires concernant le Prix SEVAL sont disponibles sur le site Web de la SEVAL : https://www.seval.ch/fr/ publications-prix-seval/prix-seval/.

\section{Literatur}

Mavrot, Céline / Hadorn, Susanne / Sager, Fritz (2019): Mapping the mix: Linking instruments, settings and target groups in the study of policy mixes, in: Research Policy Volume 48, Issue 10, December 2019, https://doi.org/10.1016/j. respol.2018.06.012.

Pawson, Ray / Tilley, Nick (1997): Realistic Evaluation, London, SAGE. 